\title{
Primary diffuse leptomeningeal gliomatosis predominantly affecting the spinal cord: case report and review of the literature
}

\author{
A Baborie, E M Dunn, L R Bridges, J M Bamford
}

\begin{abstract}
Primary leptomeningeal gliomatosis is a rare, fatal neoplastic syndrome. A 71 year old man is reported on, who after a 2 month history of back stiffness, epigastric pain, and weight loss developed visual blurring. Cranial CT and MRI studies showed no leptomeningeal enhancement. Examination of CSF 10 weeks premortem showed an increase in protein and decrease in glucose but no malignant cells. He became increasingly confused and repeated CSF examination showed inflammation and a few suspicious cells but no definitive evidence of neoplasia. He died 7 months after onset of his initial symptoms. At postmortem meningeal whitening was seen at the base of the brain and over the spinal cord. Histology disclosed diffuse leptomeningeal gliomatosis (GFAP positive, cytokeratin negative) over the brain, optic nerves, and spinal cord without parenchymal involvement. No tumour was found in internal organs. The diagnosis of primary leptomeningeal gliomatosis was not evident after cranial CT and MRI and CSF examination premortem. Suspected cases need MRI scanning of the entire neuraxis and meningeal biopsy.

(F Neurol Neurosurg Psychiatry 2001;70:256-258)
\end{abstract}

Department of Neuropathology, Algernon Firth

Building, Leeds General Infirmary, Leeds LS1 3EX, UK

A Baborie

L R Bridges

Department of Neurology, St James's University Hospital, Leeds LS 9 7TF, UK E M Dunn

J M Bamford

Correspondence to: Dr A Baborie ababorie@hotmail.com

Received 31 march 2000 and in revised form

22 September 2000

Accepted 28 September 2000 Keywords: leptomeninges; nervous system; tumour; necropsy; glioma

Primary diffuse meningeal gliomatosis (PDLG) is a rare, fatal condition characterised by widespread infiltration of the meninges by tumour apparently arising from heterotopic glial nests without evidence of tumour within the parenchyma of the brain or spinal cord. Heterotopic glial nests occur in the subarachnoid space in about $1 \%$ of unselected necropsies ${ }^{1-3}$ - most often affecting the medulla $(57 \%)^{1}$ - with a higher incidence $(25 \%)$ in patients with congenital malformations of the

At presentation, most patients complain of headache ${ }^{4}$ with signs of raised intracranial pressure or meningism. Differential diagnoses include carcinomatous meningitis and autoim- mune and inflammatory processes affecting the meninges.

We report on a patient who presented with unusual symptoms of PDLG and it was only after postmortem that the correct diagnosis was made.

\section{Case report}

A previously well 71 year old man with a 2 month history of intermittent abdominal pain, back stiffness, and weight loss was referred to a gastroenterologist in June 1998. Physical examination was normal (erythrocyte sedimentation rate $5 \mathrm{~mm} / \mathrm{h}, \mathrm{C}$ reactive protein $5 \mathrm{mg} / \mathrm{l}$ ). An endoscopy showed mild gastritis and a myeloma screen was negative. On outpatient review in mid-July he also complained of intermittent diplopia but no external ocular palsy was detected and he had normal discs on fundal examination. Contrast enhanced CT of the abdomen and head showed no relevant abnormality.

The abdominal pain worsened at the end of July 1998 but no diagnosis was made. In early August he presented with visual hallucinations, confusion, agitation, nausea, and vomiting. $\mathrm{He}$ was clearly unwell with bilateral sixth nerve palsies and papilloedema with left fundal haemorrhages. Cranial MR studies showed patent venous sinuses, a small old left temporal infarct, and ischaemic changes in addition to the known atrophy.

Over the next month he continued to lose nervous system. weight and deteriorate. Hypertensive encephalopathy was considered, as his systolic blood pressure had risen to 190 from $140 \mathrm{~mm} \mathrm{Hg}$, and porphyria was excluded. A lumbar puncture was performed. The CSF pressure was $>70 \mathrm{~cm} \mathrm{H}_{2} \mathrm{O}$, glucose was low $(0.5 \mathrm{mmol} / \mathrm{l})$; the CSF was acellular with a protein concentration of $0.5 \mathrm{mg} / \mathrm{dl}$. Further abdominal investigations were all negative.

After transfer to our unit, (4 September) he was confused and speech was rambling. Formal testing of visual fields and acuity was impossible but it was clear that he had appreciable visual impairment. Fundal examination confirmed bilaterally swollen discs with extensive haemorrhages. There was global muscle wasting and absent deep tendon reflexes, but no focal limb weakness. 

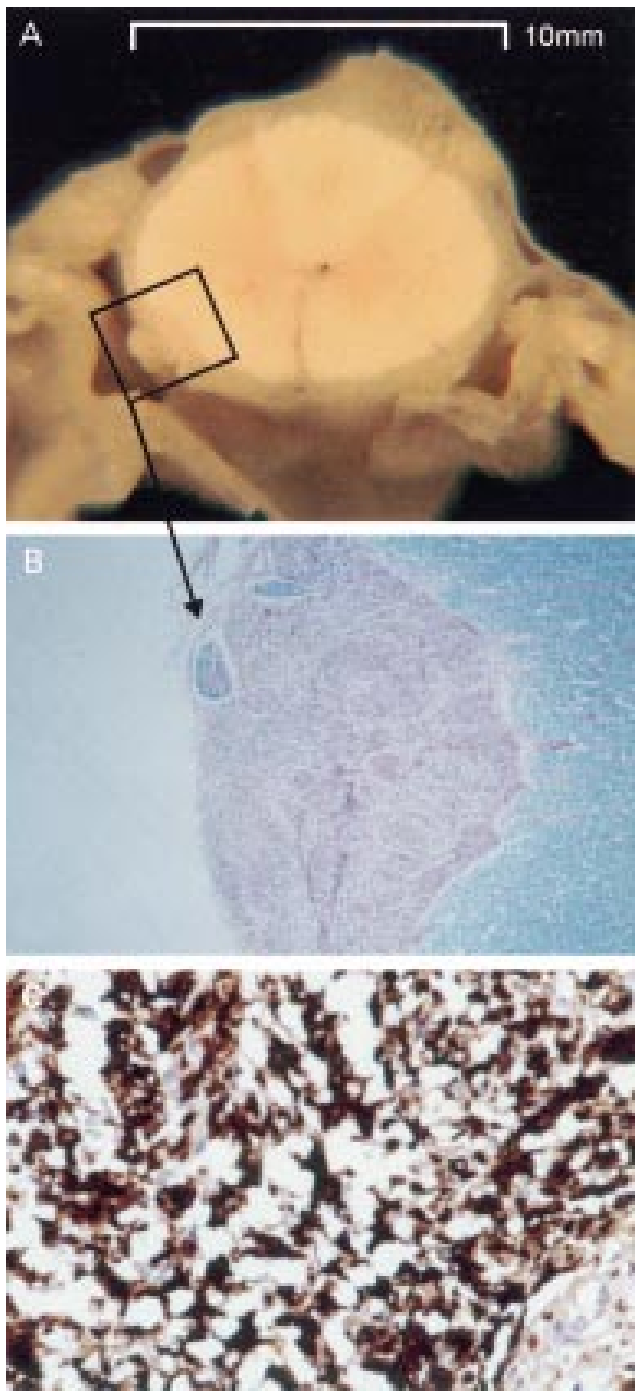

(A) Leptomeningeal tumour at T4. (B) Histology of tumour at T4 showing tumour in leptomeninges entrapping nerve roots and displacing spinal cord parenchyma (Luxol fast blue/haematoxylin and eosin originally $\times 60)$. (C) Fibrillary tumour cells were astrocytic in origin (glial fibrillary acidic protein $\times 400$ ).

Electromyography and nerve conduction studies were normal. Further MR studies were planned, but he was very restless and it was considered unsafe for him to have a general anaesthetic. Lumbar puncture was performed repeatedly (some 16 times over the 6 weeks until his death). The CSF pressure remained high $(50-80 \mathrm{~cm})$, protein just slightly increased (maximum $0.85 \mathrm{mg} / \mathrm{dl}$ ), glucose very low $(<1$ $\mathrm{mmol} / \mathrm{l})$, and the white cell count rose gradually from 0 to 16 cells/ $\mu 1$. Tuberculous and cryptococcal testing were negative. Treatment over this period was largely supportive. An empirical course of intravenous methylprednisolone had no clinical effect. He was on empirical quadruple antituberculous therapy when he died on day 4 of this treatment on 20 October 1998.

NECROPSY

The scalp, skull, dura, and venous sinuses were normal. Apart from bronchopneumonia no significant abnormality was found outside the nervous system. The brain weighed $1740 \mathrm{~g}$ and the leptomeninges showed focal areas of whitening over the vertex and base of the spinal cord with thickening maximal $(2.5 \times 2 \times 1 \mathrm{~mm})$ at $\mathrm{T} 4$ (figure $\mathrm{A}$ and $\mathrm{B}$ ).

\section{MICROSCOPICAL EXAMINATION}

Histology showed leptomeningeal infiltration by a diffuse infiltrating astrocytoma over the brain, spinal cord, and optic nerves. Spindle shaped tumour cells with fibrillary processes and pleomorphic, vesicular nuclei were seen extensively within the reticulin meshwork of the leptomeninges. Tumour cells (figure C) expressed GFAP (polyclonal antibody, DAKO, 1:200). The proliferation rate (proliferation marker Mib-1 or Ki-67, monoclonal antibody, Immunotec, 1:50) was focally up to $20 \%$ (tumour at T4) and varied in other areas down to $3 \%$. Tumour cell nuclei were p53 negative (monoclonal antibody, Novo Castra, clone DO7, 1:50 and 1:20). There was no discrete focus of tumour within the parenchyma of the brain or spinal cord. This was therefore considered a case of primary diffuse leptomeningeal gliomatosis.

\section{Discussion}

As well as being a rare diagnosis the case illustrates the wide constellation of symptoms and signs that PDLG may manifest. This is the seventh reported case of PDLG predominantly affecting the cord..$^{5-10}$ Of the 21 cases of PDLG reported since 1957, 18 were verified at necropsy.

An increased CSF protein combined with low CSF glucose, especially in the presence of a high CSF cell count and raised intracranial pressure, as in our case, indicates a possible neoplastic process, and has been noted in most of the reported cases of PDLG. The raised CSF pressure in our case was most likely due to tumour mediated obstruction of CSF drainage into spinal nerve roots, ${ }^{11}$ as the bulk of the tumour was spinal rather than cerebral and the arachnoid granulations were free of tumour. The increased CSF protein concentration may also have contributed to the high pressure gradient. ${ }^{5}$

The signs in the present case did not initially suggest the need for spinal MRI examination. In retrospect that might have been helpful in obtaining a diagnosis although given the small size of the lesion at $\mathrm{T} 4$ at necropsy it seems likely that this would not have been enhanced even with MRI earlier in the illness ${ }^{12}$; MRI enhancement of the meninges has been noted in only a few reports. ${ }^{5}{ }^{8-10}$

In reported cases of PDLG without a clear premortem diagnosis (as in our patient) antituberculous treatment is almost always tried. Patients treated additionally with radiation or chemotherapy survive slightly longer than with drug treatment alone ${ }^{13}$; one patient even improved $^{9}$ and a further patient went into complete remission for 15 months. ${ }^{14}$ If the CSF showed atypical cells ${ }^{51516}$ and the patient's therapy included irradiation ${ }^{516}$ remission or clinical improvement is reported (by contrast with treatment with chemotherapy alone).${ }^{15}$ Survival is also associated with different factors such as the World Health Organisation (WHO) grade of the 
tumour, complicating lesions such as infarcts (due to vascular compression by adventitial tumour mass), and the site of the lesion-for example, involvement of vital centres. ${ }^{10}$

The overall poor prognosis (comparable with gliomatosis cerebri) suggests a worse biological behaviour than predicted by WHO grade of the biopsy in many cases.

As the bulk of the PDLG as in this case may be spinal rather than cerebral, spinal as well as cranial MRI should be considered in suspected cases. In PDLG as in gliomatosis cerebri, MRI is superior to $\mathrm{CT}$ in detecting diffusely infiltrating neoplastic astrocytes. ${ }^{917}$

We thank Steve Toms and Michael Todd for photographic assistance.

1 Cooper IS, Kernohan JW. Heterotopic nests in the subarachnoid space: histologic characteristics, mode of origin and relation to meningeal gliomas. $\mathcal{F}$ Neuropathol Exp Neurol 1951;10:16-21.

2 Freeman W. Cortical heterotopia in the pontile meninges. Arch Pathol 1926;2:352-4.

3 Wolbach SB. Congenital rhabdomyoma of the heart. Report of a case associated with multiple nests of neuroglia tissue in the meninges of the spinal cord. F Med Res 1907;16:495519

4 Dietrich PY, Aapro MS, Rieder A, et al. Primary diffuse leptomeningeal gliomatosis (PDLG): a neoplastic cause of chronic meningitis. F Neurooncology 1993;15:275-83.

5 Kobayashi M, Hara K, Nakatsukasa $M$, et al. Primary spinal leptomeningeal gliomatosis presenting visual disturbance as the initial symptom. Acta Neurochir (Wien) 1996;138:480-1.
6 Park JS, Van den Noort S, Kim RC, et al. Primary diffuse leptomeningeal gliomatosis with signs of increased intracranial pressure and progressive meningeal enhancement on MRI. F Neuroimag 1996:6:250-4.

7 Rogers LR, Estes ML, Rosenbloom SA, et al. Primary leptomeningeal oligodendroglioma. Neurosurgery 1995;36: $166-9$.

8 Giordana MT, Bradac GB, Pagni CA, et al. Primary diffuse leptomeningeal gliomatosis with anaplastic features. Acta Neurochir (Wien) 1995;132:154-9.

9 Leproux F, Melanson D, Mercier C, et al. Leptomeningeal gliomatosis: MR findings. Fournal of Computer Assisted Tomography 1993;17:317-20.

10 Ramasy DA, Goshko V, Nag S. Primary spinal leptomeningeal astrocytoma. Acta Neuropathol 1990;80:338-41.

11 Weller RO. Pathology of cerebrospinal fluid and interstitial fluid of the CNS: significance for Alzheimer disease, prion disorders and multiple sclerosis. 7 Neuropathol Exp Neurol disorders and mult

12 Kastenbauer S, Danek A, Klein W, et al. Primary diffuse leptomeningeal gliomatosis: unusual MRI with nonenhancing nodular lesions on the cerebellar surface and spinal leptomeningeal enhancement. I Neurol Neurosurg Psychiatry 2000;69:385-8.

13 Witham TF, Fukui MB, Meltzer CC, et al. Survival of patients with high grade glioma treated with intrathecal thiotriethylenephosphoramide for ependymal or leptomeningeal gliomatosis. Cancer 1999;86:1347-53.

14 Beauchesne P, Pialat J, Duthel R, et al. Aggressive treatment with complete remission in primary diffuse leptomeningeal gliomatosis. F Neuro-Oncol 1998;37:161-7.

15 Singh M, Corboy JR, Stears JC, et al. Diffuse leptomeningeal gliomatosis associated with multifocal CNS infarcts. Surg Neurol 1998;50:356-62.

16 Kithara M, Katakura R, Wada T, et al. Diffuse form of primary leptomeningeal gliomatosis. $\mathcal{f}$ Neurosurg 1985;63: 283-7.

17 Shin YM, Chang KH, Han MH, et al. Gliomatosis cerebri: comparison of MR and CT features. AfR Am f Roentgenol 1993;161:859-62. 